\title{
Breastfeeding Support and Counseling: A Practical Guide for the General Practitioners in Outpatient Clinics
}

\author{
Kammi Yap Sayaseng, DNP, RN, PNP-BC, IBCLC ${ }^{1}$ \\ ${ }^{1}$ California State University, Fresno, School of Nursing and Valley Children's Medical Group, United States \\ Correspondence: Kammi Yap Sayaseng, DNP, RN, PNP-BC, IBCLC, California State University, Fresno, School \\ of Nursing and Valley Children's Medical Group, United States.
}

Received: August 13, 2018

Accepted: August 28, 2018

Online Published: August 31, 2018

doi:10.20849/ijsn.v3i3.459

URL: https://doi.org/10.20849/ijsn.v3i3.459

\begin{abstract}
Many studies have cited the need for more time and adequate training in breastfeeding (BF) support and counseling for health care professionals in the outpatient setting. As a pediatric nurse practitioner, primary care provider (PCP), and an International Board Certified Lactation Consultant (IBCLC), this dilemma is well understood. With the establishment of baby-friendly hospitals, BF mothers receive the much needed BF counseling and support before going home. However, the length of hospital stays for mothers with healthy, full-term newborns (NBs) has dramatically decreased with the advancement of technologies in the healthcare field. Grassley, Schleis, Bennett, Chapman and Lind (2014) found that 38\% of NBs whose mothers planned to exclusively breastfeed (BF) received formula before hospital discharge in their retrospective review of 302 hospital charts. Professional BF support in the outpatient setting is inadequate or insufficient. The first 2 weeks of life are crucial to the continuation of exclusive breastfeeding (EBF) or its termination. Most NBs are seen for hospital discharge follow-up within 5 days. PCPs have the ideal opportunity to address BF support and/or counseling at this crucial time with BF mothers and their support person(s) to promote, sustain, and maintain EBF.
\end{abstract}

Keywords: breastfeeding, breastfeeding support, breastfeeding counseling, general practitioners, outpatient clinic, international board certified lactation consultant, breastfeeding barriers

\section{Introduction}

There are many barriers to successful BF and this article aims to address, as well as provide clinical pearls to, some of the common ones seen in the outpatient setting. In no particular order, they are (a) absent or inadequate provider training in the subject of BF support and counseling; (b) provider time constraint; (c) mother's cracked, sore, and/or bleeding nipples; and (d) language barrier. General practitioners in outpatient settings have expressed that inadequate training/lack of education in BF support and counseling prevent much-needed services to BF mothers in need. Over $71 \%$ of both practicing pediatricians and OB-Gyns reported having little or no BF education or training (Sriraman \& Kellams, 2016).

Even for providers adequately trained in lactation support and counseling, time constraint is a key barrier to providing adequate services in the outpatient setting. In the United States, most PCPs have 15 minutes or less per patient in an outpatient clinic. This is hardly enough time to sufficiently address patient problems. BF support and counseling take anywhere from 15 minutes to 1 hour for a mom and baby. Ideally, it is best to have someone who is specifically trained in BF, an IBCLC, included in the healthcare team. Increased BF success has been associated with an increase in professional lactation support, especially when this support is provided by an IBCLC (Dennison, Nguyen, Gregg, Fan, \& Changning, 2016). Including an IBCLC can directly address provider training inadequacy and time constraint as barriers to successful BF in outpatient clinics or settings. Including an IBCLC in the healthcare team can pose problems as well, such as availability of resource personnel, budget constraints, etcetera.

Some barriers to successful BF or EBF are inherent, such as with pain from cracked, sore, and/or bleeding nipples secondary to improper latch while BF. This problem can be addressed by observing the mother BF her NB while in the clinic with the provider. As for language barriers, it is critical that providers have the proper resources. Patient satisfaction, adherence, and health outcomes have been linked to clinician-patient communication. Clinicians who disregard the importance of sociocultural differences [including limited English 
proficiency (LEP)] almost certainly provide lower quality care (Batencourt, Gree, \& Carillo, 2018).

\section{Method}

\subsection{Case Study 1}

\subsubsection{Patient History}

A 5-day-old NB male presented to the outpatient pediatric clinic with his parents for a NB check following hospital discharge. Patient was born full-term via normal spontaneous vaginal delivery (NSVD) without complications and was discharged home after 24 hours; EBF at hospital discharge.

\subsubsection{Parent History}

The mother indicated that they wanted to do EBF for their NB. This was the couple's first baby. Parents' native language was Lao. Both were in their mid-30s and recent migrants to the U.S. Both parents had limited English-speaking skills. They did not have any local family support.

\subsubsection{Chief Complaint}

Parents reported that the baby cried a lot. When put to the breast, "he would get mad and not feed." This led the parents to give the NB some formula feedings. They reported that the NB drank from the bottle "better" than from mom's breasts. This had been going on since they left the hospital.

\subsubsection{Assessment and Solutions}

The author saw the patient and his parents the day they presented to the clinic. The author is a PNP, an IBCLC, and is fluent in five different languages, one of which is Lao. The parents were ecstatic to see a provider who could communicate in their native language. When the author asked about feeding history (how often and how long on the breast, etc.), the parents reported that the baby was put to the breast exactly every 3 hours, as told to them at their hospital discharge. When asked why they thought their baby "cried a lot," hunger was not one of their answers. When asked if they fully understood what was relayed to them at discharge, they admitted "no."

\subsubsection{Clinical Pearls}

This case study illustrates some key complications: (a) language barrier, (b) cultural incompetence, and (c) inadequate hospital discharge instructions. Most Southeast Asian people (including Lao) respect people in authorative positions - in this case, the health care providers. When they were told to feed the baby "every 3 hours," they fed the baby "every three hours," as instructed. It is wise to have patients repeat back the instructions heard to ensure they understand them completely and thoroughly. Offer an interpreter if English is not a patient's primary language. With BF, parents should be told to "breastfeed on demand; when the baby wants to feed," not every 3 hours. Hunger cues should have been taught and reinforced at hospital discharge.

Healthcare providers must see and acknowledge the wide diversity of ethnicities and cultures among their patients and provide genuinely competent cultural care.

\subsection{Case Study 2}

\subsubsection{Patient History}

Fourteen-day-old Caucasian male born full term via NSVD without any complications and was discharged home after 24 hours. Patient was EBF.

\subsubsection{Parent History}

Both parents were in their early 30s and both were college graduates. This was their first baby. Patient's maternal great aunt was a pediatrician and lived close by the family.

\subsubsection{Chief Complaint}

Patient and parents presented to the pediatric outpatient clinic for "follow-up weight." Baby had not been gaining appropriately at the expected rate of 25 to 30 grams per day and was still not back at his birth weight at 14 days of life.

\subsubsection{Assessment and Solution}

The author saw the patient at this visit. When asked about BF history, mom reported that she was "doing great." She stated that she breastfed the baby "on demand." When asked if she felt she was producing milk (her breasts felt full before the baby fed and empty after feeding), she stated "not really." When asked if she had any problems BF her baby, she stated "No. I think I'm doing fine." The provider asked for permission to assess the mother's breasts (to check for inverted nipples and/or any breast deformities) and "observe" her BF techniques. 
On assessment, it was noted that both of the mother's nipples were cracked, but not actively bleeding. It was also noted that the mother put her baby to the breast while the baby's mouth was not completely open. The baby's top and bottom lips were not flanged out after latching onto the breast to feed. The provider asked if she experienced any nipple or breast pain with this feeding and she replied, "Yes. Actually, it hurts me every time I feed him. Nevertheless, I just kept going because it was something that I have to do for my baby. I was not going to let pain stop me from breastfeeding him." This case was resolved when the provider spent more than 30 minutes working with both parents on proper latching. After a few attempts, the mother successfully latched her baby onto her breast for feeding with very minimal nipple pain. Both parents, especially the mother, were visibly grateful for the time spent helping them with proper latch and technique. The mother's aunt was a local pediatrician and stated that she could help support her BF technique at home. The patient did not get admitted to the hospital, as the baby's slow weight gain was attributed to improper latch, as all other pathological problems had been ruled out. The provider telephoned the parents that same evening and learned that the mom was not experiencing any nipple pain during or after BF. They were brought in to the clinic the next day for follow-up weight. At the last visit, the NB had gained more than 35 grams per day and was above his birthweight then.

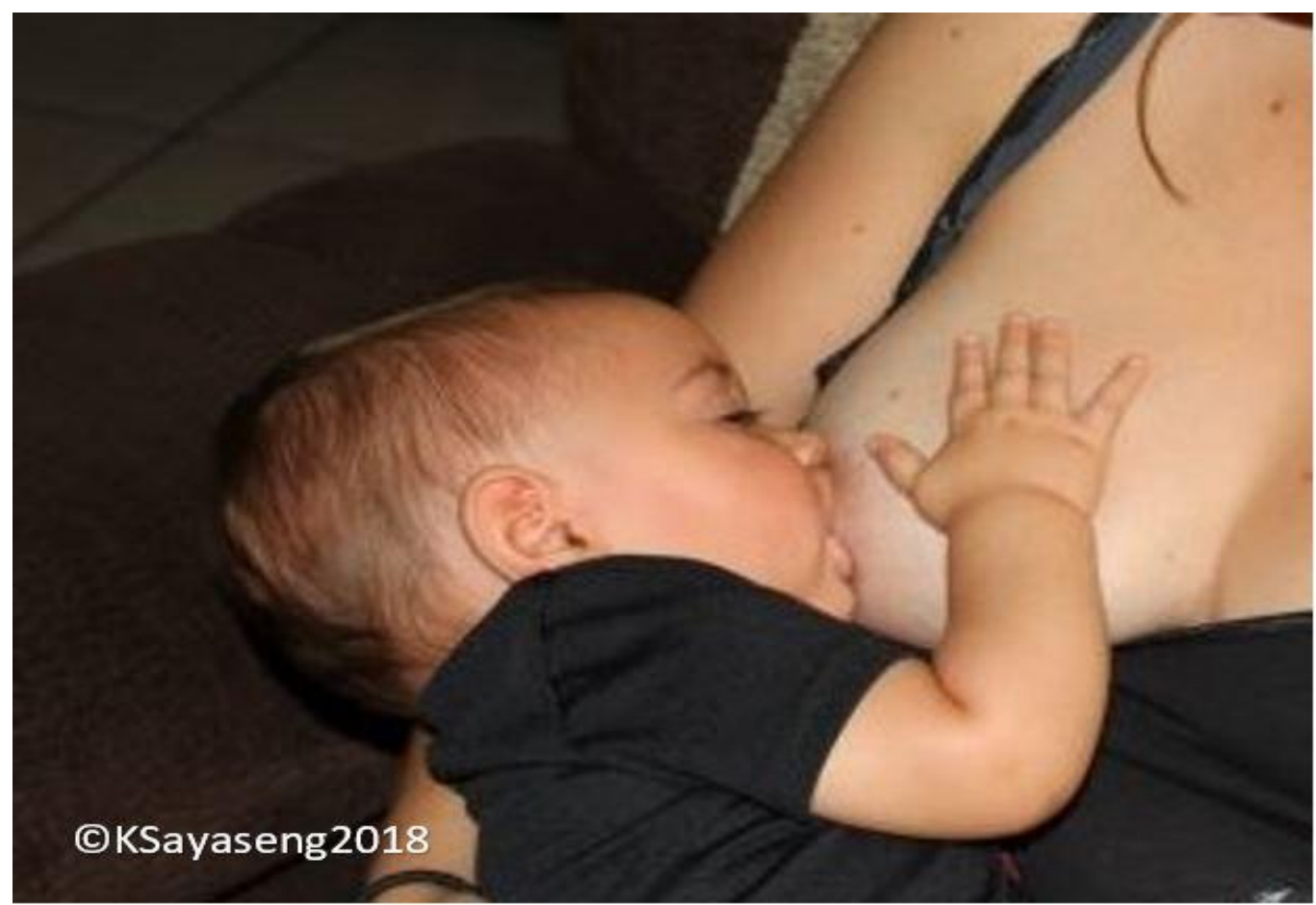

Figure 1. Proper breastfeeding latch with baby's top and bottom lips flanged out

\subsubsection{Clinical Pearls}

In this case study, both parents were educated and had BF support at home. The mother felt that it was her "duty and responsibility to breastfeed" her NB, no matter the cost. This case demonstrates how a provider should never stop at asking, "How is breastfeeding coming along for you and your baby?" This mother answered that she was "doing great," when, in fact, she was experiencing nipple pain due to inappropriate latch during feedings. Always ask for permission to check the mother's breasts and observe her BF technique in clinic if the baby is not gaining proper weight within the first 2 weeks of life. Healthy term NBs should regain their birthweights by day 10 to 14 of life. If not, the provider must consider all possible factors in determining the cause of the slow weight gain. In this case, the NB had no medical problems other than mild jaundice.

\section{Conclusions}

The majority of parents decide to BF before or during pregnancy. Providing prenatal counseling on BF at obstetrics visits would be ideal. Over $71 \%$ of both practicing pediatricians and OB-Gyns felt they had little or no BF education or training and felt "uncomfortable telling a mother how to feed a baby" (Sriraman \& Kellams, 2016). Li et al. (2014) found that in addition to educating hospital staff to improve BF related knowledge and 
implementing hospital related BF policies, ensuring continuity of prenatal and postnatal BF education and support may improve newborn BF rates. Schreck et al. (2017) also found that prenatal BF education and ongoing postdischarge support are needed to improve BF continuation.

Cottrell and Detman (2013) noted one mother's comment about BF. "But ... when I was discharged ... she wouldn't nurse at all that day, so, sometime late in that evening I just went on and gave her the formula that they had provided ... and I almost cried at the way she sucked that bottle down, because I'm like, I sat here and starved my child all day." Lactogenesis involves a series of cellular changes where mammary epithelial cells are converted from a nonsecretory state to a secretory state. There are two phases/stages to lactogenesis: (a) Secretory initiation, also known as Stage I, or Lactogenesis I, which occurs in the second half of a pregnancy; and (b) Secretory activation, also known as Stage II, or Lactogenesis II, which occurs after delivery. Breast swelling, or feelings of breast fullness, are followed by milk production after delivery. This typically occurs two to three days postpartum, but in some cases will take as many as 7 days or longer. Occasionally it will happen before delivery (Schanler \& Potak, 2018).

Young age, lower income, less maternal education, unmarried status, and need to return to work/school are some of the socioeconomic deterrents to EBF (Besore, 2014; Sriraman \& Kellams, 2016). According to Jones, Power, Queenan and Schulkin (2015) and Sriraman and Kellams (2016), racial and ethnic minority, infant formula samples, and hospital staff trainings and hospital policies with early BF are additional barriers to EBF.

As demonstrated in the second case study, the parents had none of the stated socioeconomic deterrents and still struggled with BF. Contrary to popular opinion, BF, while beautiful, is no easy task for most mothers. The decision to BF ultimately belongs to the mother, but her familial and cultural traditions will profoundly influence her decision-making process. Within this process are the fathers, who generally feel incapable of helping their wives with BF. However, they can help by setting up and caring for baby while mom gets ready to BF. They can also care for baby while mom takes a needed daytime nap.

\section{Acknowledgements}

The author wishes to acknowledge baby Joseph Ray Villagomez, Jr. and his parents Jennah and Joseph Ray Villagomez for the photo.

\section{References}

Batencourt, J.R., Green, A.R., \& Carrillo, J.E. (2018). Cross-cultural care and communication. Retrieved from https://www.uptodate.com/contents/cross-cultural-care-and-communication?search=interpreter\%20service \&sectionRank=1\&usage_type=default\&anchor=H22\&source=machineLearning\&selectedTitle $=1 \sim 150 \&$ dis play_rank=1\#H22

Besore, C.T. (2014). Barriers to breastfeeding for Hispanic mothers. Breastfeeding Medicine, 9(7), 352-354. https://doi.org/10.1098/bfm.2014.0090

Cottrell, B.H., \& Detman, L.A. (2013). Breastfeeding concerns and experiences of African American mothers. Maternal Child Nursing, 38(5), 297-304. https://doi.org/10.1097/NMC.0b013e31829a5606

Dennison, B.A., Nguyen, T.Q., Gregg, D.J., Fan, W., \& Changning, X. (2016). The impact of hospital resources and availability of professional lactation support on maternity care: Results of breastfeeding survey 2009-2014. Breastfeeding Medicine, 11(9). https://doi.org/10.1089/bfm.2016.0072

Grassley, J.S., Schleis, J., Bennett, S., Chapman, S., \& Lind, B. (2014). Reasons for initial formula supplementation of healthy breastfeeding newborns. Nursing for Women's Health, 18(3). https://doi.org/10.1111/1751-486X.12120

Jones, K.M., Power, M.L., Queean, J.T., \& Schulkin, J. (2015). Racial and ethnic disparities in breastfeeding. Breastfeeding Medicine, 10(4).

Li, C.M., Li, R., Ashley, C.G., Smiley, J.M., Cohen, J.H., \& Dee, D.L. (2014). Associations of hospital staff training and policies with early breastfeeding practices. Journal of Human Lactation, 30(1), 88-96. https://doi.org/10.1177/0890334413484551

Schanler, R.J., \& Potak, D.C. (2018). Physiology of lactation. Retrieved from https://www.uptodate.com/contents/physiology-of-lactation?search=lactogenesis\&source=search_result\&se lectedTitle $=1 \sim 13 \&$ usage_type $=$ default $\&$ displayrank $=1$ 
Schreck, P.K., Solem, K., Wright, T., Schulte, C., Ronnisch, K.J., \& Szpunar, S. (2017). Both prenatal and postnatal interventions are needed to improve breastfeeding outcomes in a low-income population. Breastfeeding Medicine, 12(3), 146-148. https://doi.org/10.1098/bfm.2016.0131

Sriraman, N.K., \& Kellams, A. (2016). Breastfeeding: What are the barriers? Why women struggle to achieve their goals. Journal of Women's Health, 25(7), 714-722. https://doi.org/10.1089/jwh.2014.5059

\section{Copyrights}

Copyright for this article is retained by the author(s), with first publication rights granted to the journal.

This is an open-access article distributed under the terms and conditions of the Creative Commons Attribution license (http://creativecommons.org/licenses/by/4.0/). 